\title{
Progress on All Solid State Deep-ultraviolet Laser with $\mathrm{KBe}_{2} \mathrm{BO}_{3} \mathrm{~F}_{2}$ Crystal
}

\author{
Qinjun PENG, ${ }^{1}$ Xiaoyang WANG,${ }^{2}$ Yong ZHOU,${ }^{1}$ Yong ZHU, ${ }^{2}$ Chengming LI, ${ }^{1}$ Zhanggui HU, ${ }^{2}$ \\ Dafu CUI, ${ }^{1}$ Chuangtian CHEN, ${ }^{2}$ and Zuyan $\mathrm{XU}^{1}$ \\ ${ }^{1}$ Laboratory of Optical Physics, Institute of Physics, Chinese Academy of Sciences, P.O. Box 603, \\ Beijing 100080, China, \\ ${ }^{2}$ Beijing Center for Crystal R\&D, Technical Institute of Physics and Chemistry, Chinese Academy of \\ Sciences, P.O. Box 2711, Beijing 100080, China
}

(Received January 25, 2008)

\begin{abstract}
General review for the progress on all solid state deep ultraviolet (DUV) laser with a $\mathrm{KBe}_{2} \mathrm{BO}_{3} \mathrm{~F}_{2}(\mathrm{KBBF})$ crystal was given in the paper. The frequency-conversion characteristics of KBBF crystal and the comparison between this crystal and other borate nonlinear optical crystals were simply described. A special prism coupling technique (PCT) for DUV laser generation and some excellent experimental results from the application of this crystal were commented. At last, we believe that the output power and conversion efficiency could be greatly increased in the future if the thicker KBBF crystal with good optical quality is grown and the application technique of solid state DUV laser is further developed.
\end{abstract}

Key Words: DUV, KBBF, Solid state laser, Nonlinear optics, SHG

\section{Introduction}

Deep ultraviolet (DUV) sources can be applied to many areas, such as semiconductor photolithography, micro-machining, laser spectroscopy, photoemission spectroscopy and photochemical synthesis. ${ }^{1)}$ The DUV sources can be synchrotron light sources, gas-discharge sources or laser sources. Synchrotrons offer unparalleled flexibility in wavelength tenability $(1-1000 \mathrm{eV})$, but they cause low energy resolution $(10 \mathrm{meV})$ and small photon flux $\left(10^{12}-10^{13}\right.$ photons/Second). Although gas-discharge sources offers better energy resolution $(1.2 \mathrm{meV})$, it has drawbacks like large beam size $(2 \sim 3 \mathrm{~mm})$ and small photon flux $\left(10^{12}\right.$ photons/second). The DUV lasers, including the excimer laser, solid state laser and so on, are highly desirable for many applications. The excimer lasers can emit coherent light with a high average power output, however, their wavelength tunability and beam quality were poor. So, the compact, efficient solid-state lasers are more attractive, because of their narrower spectral bandwidth, better beam quality and easier maintenance etc. In the past, the solid state DUV lasers are generated by use of sum-frequency method, however, the need for two beams which are one short wavelength and the other long wavelength can be an inconvenience. So far, only $\mathrm{KBe}_{2} \mathrm{BO}_{3} \mathrm{~F}_{2}$ (KBBF) crystals can be used to achieve the DUV laser light through second harmonic generation (SHG). Similarly, the shorter wavelength can be also obtained by the sum-frequency generation (SFG) method in KBBF crystal. In the report, we made a general review for the progress on high power and high efficient DUV laser with a KBBF crystal.

\section{Characteristics of KBBF Crystal}

KBBF crystal ${ }^{2)}$ was first synthesized by the former Soviet
Union scientists in 1968. The crystal structure was re-determined by $\mathrm{C}$. Chen, and the results showed that $\mathrm{KBBF}$ crystallizes in the space group R32 (not C2). KBBF is an excellent nonlinear optical (NLO) crystal with relatively larger SHG coefficients, wide transparent region from 152 to 3664 $\mathrm{nm}$, moderate birefringence, out of hygroscopy, good optical uniformity with $\delta \mathrm{n} \approx 10^{-4} / \mathrm{cm}$, high damage threshold as high as $4 \times 10^{12} \mathrm{~W} / \mathrm{cm}^{2}$ at $532 \mathrm{~nm}$ with 7 -ns pulse width and $10 \mathrm{~Hz}$, high thermal conductivity of $\sim 2.5 \mathrm{~W} / \mathrm{mK}$, a wide band gap of $\sim 8.2 \mathrm{eV}$. The melting point of KBBF is estimated to be above $1100 \mathrm{C}$ and it volatilizes severely and decomposes above $800 \mathrm{C}$. Therefore, KBBF crystals is mainly grown from a flux melt, not hydrothermal method above $800{ }^{\circ} \mathrm{C}$. Crystals showed a plate-like growth habit due to layered structure of KBBF. At present, KBBF can be grown with its thickness restricted to 3 $\mathrm{mm}$ only, the length along the $\mathrm{z}$ axis has not exceeded $2.5 \mathrm{~mm}$ till now. Although the hydrothermal method below $750{ }^{\circ} \mathrm{C}$ condition can be used to grow the large size $\mathrm{KBBF}$ of $\sim 10 \mathrm{~mm}$, the Phi degree was confusable at present. The plate-like crystals were still too thin to be cut along the phase-matching direction. A special prism coupling technique (PCT) ${ }^{3)}$ was invented to solve the question in cutting the crystal. The invention was a sandwich structure and KBBF crystal is placed in between two prisms (UV fused silica or $\mathrm{CaF}_{2}$ crystal). The two interfaces between the KBBF and $\mathrm{CaF}_{2}$ (or fused silica) were brought into optical contact for reducing the interface losses of the laser beam, or the interfaces were filled with matching oil or deionized water. When the fundamental wave is input along the normal direction of the prism, the angle of refraction in KBBF is equal to the apex angle of the prism. The phase matching of the wavelength can be automatically achieved in KBBF when the special fundamental wave is input along the normal direction and the polarization direction of the fundamental wave is along the $a$ 

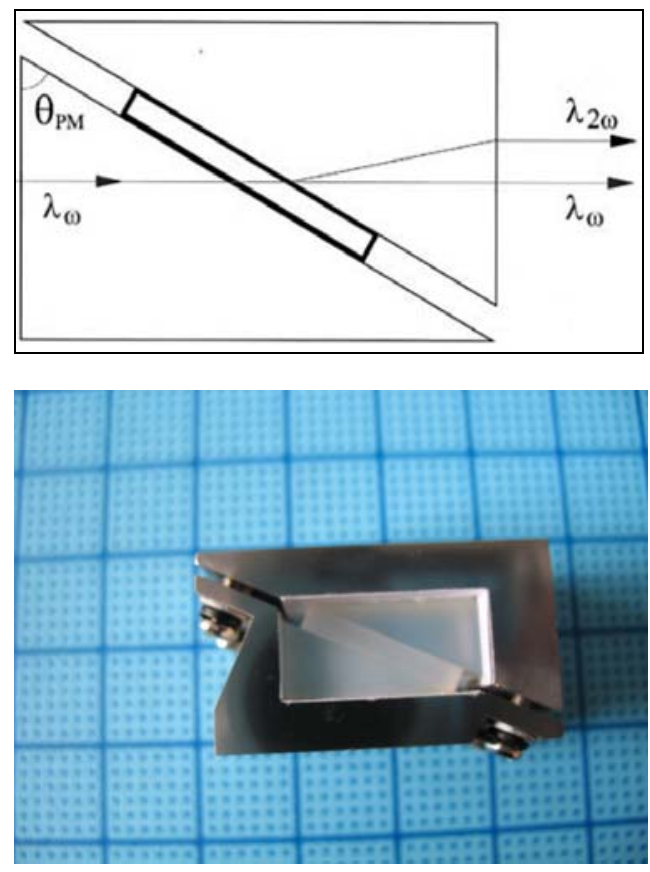

Fig.1 Scheme of the KBBF-PCT and the optical contact prism-coupled setup.

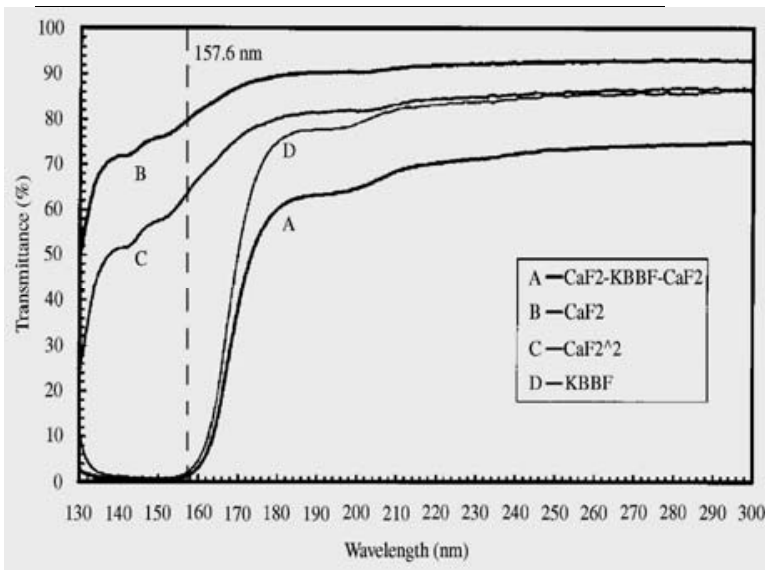

Fig.2 Curves A, B, C, and D represent the transmission spectra of KBBF crystal plus two $\mathrm{CaF}_{2}$ prisms, a single $\mathrm{CaF}_{2}$ prism, two $\mathrm{CaF}_{2}$ prisms, and a single $\mathrm{KBBF}$ crystal, respectively

axis of KBBF, if the apex angle of the prism is equal to the phase-matching angle of KBBF at a special wavelength. It can be seen that this crystal can produce very short wavelength from second to harmonic output from the above narration. The KBBF-PCT setup and the transmittance of KBBF device were shown in Fig. 1 and 2, respectively.

\section{All Solid State DUV Laser Generation}

3.1 Phase Matching of KBBF and Other Borate Crystals ${ }^{3)}$ So far, only KBBF crystal can be use to obtain the DUV laser below $200 \mathrm{~nm}$ by the SHG generation from Fig 3. In
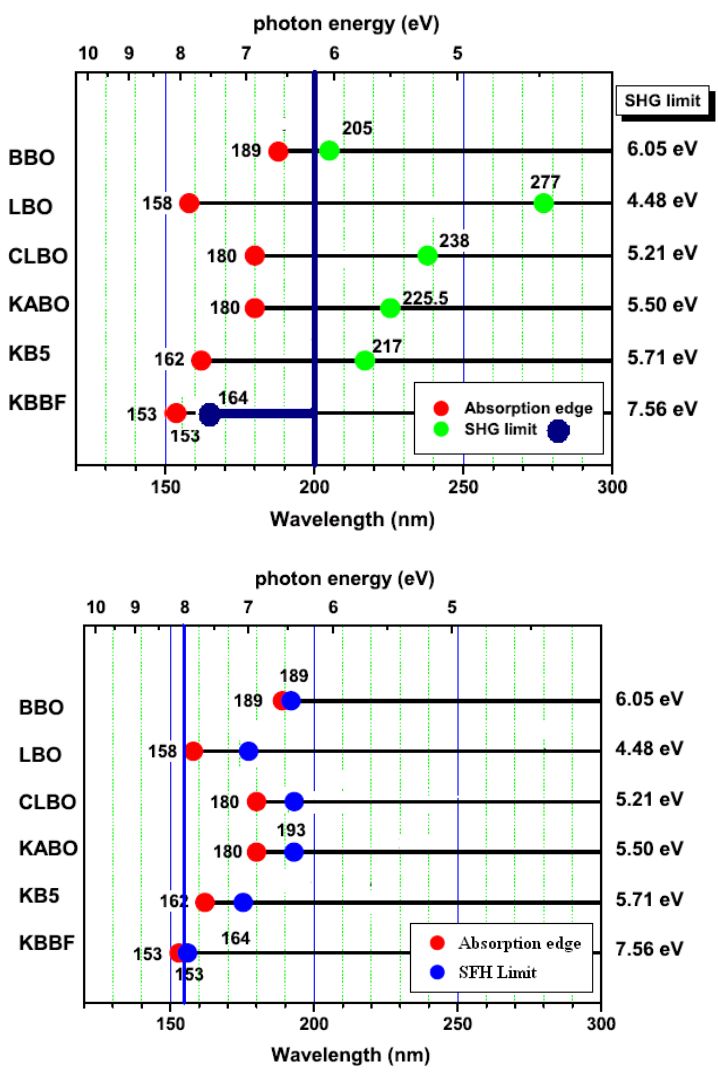

Fig.3 SHG and SFG limit for some typical borate crystals

addition, although some crystals such as LBO, BBO, CLBO, $\mathrm{CBO}, \mathrm{SBBO}, \mathrm{TBO}, \mathrm{KABO}, \mathrm{BABO}, \mathrm{KB} 5$ etc, also can generate the DUV through SFG technique, at present, the obtained wavelength is shortest in DUV region by KBBF SFG technique.

The birefringence of LBO is only about 0.045 , which is too small to achieve deep-UV harmonic generation. The same situation also occurs in the other borate crystals of the LBO family such as CBO and CLBO. It is known that even though CBO, CLBO KB5 can achieve DUV output, two different wavelength beams must be included as input, which is obviously not convenient for practical applications. From Fig. 3 , we immediately knew that the capability of BBO to produce the DUV coherent light below $200 \mathrm{~nm}$ was limited by the absorption edge $(189 \mathrm{~nm})$. The SBBO structure has macroscopic order but microscopic disorder, which could be a reason why the optical uniformity of SBBO is very poor. As a result, the phase-matching angles of the crystal at the different wavelengths cannot be determined accurately till now, which heavily limits its applications in various frequency-conversion devices. TBO crystal seems to have the same structure problem as SBBO. Conversely, the space structure of KABO can be determined exactly, so high optical quality crystals of $\mathrm{KABO}$ in large size can be grown. KABO can also achieve fourth and fifth-harmonic generation of a Nd:YAG laser and 193-nm wavelength output with sum-frequency generation. In addition, this crystal is not hygroscopic and has good mechanical properties for cutting, polishing, and coating. Therefore, KABO is a good potential UV and deep-UV NLO crystal, and further research is deserved. The BABO crystal 
should have the same optical properties as $\mathrm{KABO}$, but a crystal large enough have not been yet obtained to measure its linear and nonlinear optical properties.

3.2 DUV Laser Generation in Typical Borate Crystals ${ }^{3)}$

LBO was used to generate the DUV in the range of $172.7-187 \mathrm{~nm}$ by phase-matched sum-frequency mixing of the Ti:sapphire's $(1 \mathrm{kHz} 0.3 \mathrm{~mJ}, 740-850 \mathrm{~nm}, 150-200 \mathrm{fs})$ fourth harmonic $(\omega+3 \omega)$ and a parametrically generated infrared pulse which was generate by an optical parametric generator/amplifier scheme with the same Ti:sapphire laser as the pump source. ${ }^{4)}$ A similar experimental setup as LBO was used to generate the DUV from 172 down to $166 \mathrm{~nm}$ in a KB5 crystal. ${ }^{5)}$ In addition, the KB5 was the first borate crystal to achieve 194-nm coherent light output with the sum-frequency mixing method $(792 \mathrm{~nm}+515 \mathrm{~nm} \rightarrow 194 \mathrm{~nm})$. $177.4 \mathrm{~nm}$ DUV laser in KB5 was provided by the harmonic generation of a $\mathrm{Nd}$ :YAG laser $(\omega+5 \omega)$. BBO crystal was the first borate crystal which obtained the $193 \mathrm{~nm}$ effective output by the SFG of a linearly polarized picosecond $\mathrm{KrF}$ excimer laser at $248.5 \mathrm{~nm}$ and a tunable dye laser at $950-800 \mathrm{~nm}$. Recently, The fourth-harmonic generation $(\omega+3 \omega)$ of a Ti:sapphire laser in BBO was used to obtained 22-mW power output at 193.5 $\mathrm{nm}$. Using the same method in CBO, 193.3nm was obtained the effective output. Also, CBO was used to generate 193-nm coherent light output through a sum-frequency mixing process $(2.0 u m+213 \mathrm{~nm}$ to $193 \mathrm{~nm})$, meanwhile, over $5 \mathrm{~mJ} /$ pulse energy has been obtained. CLBO crystal was used to generate $193 \mathrm{~nm}$ output though an eighth-harmonic generation $(\omega+7 \omega$ process $)$ of an Er-doped fiber amplifier system. Thereinto, the $2 \omega, 3 \omega$, and $4 \omega$ harmonics were produced in LBO crystal, BBO was used to SFG $(3 \omega+4 \omega)$ for $7 \omega$ generation. KABO crystal can generate $193 \mathrm{~nm}$ with a SFG procedure $(1064 \mathrm{~nm}+236 \mathrm{~nm}$ to $193.2 \mathrm{~nm})$.

\subsection{DUV Generation in KBBF Crystal}

In order to produce wavelengths below $200 \mathrm{~nm}$, only KBBF so far has enough birefringence and sufficient transparency range to achieve directly fourth or fifth-harmonic generation of a Ti: sapphire laser. It also can be used to generate the fourth harmonic of the entire tunable spectral region of the Ti: sapphire laser. The crystal can further achieve sixth-harmonic generation of a Nd:YVO4 (or Nd:YAG) laser $(3 \omega+3 \omega$ procedure) to produce $177.3-\mathrm{nm}$ coherent light output. The shortest SHG wavelength of KBBF is $162 \mathrm{~nm}$ and the shortest theoretical SHG wavelength obtained experimentally so far is $170 \mathrm{~nm}^{6}{ }^{6}$ In addition, at present, the shortest wavelength has been obtained by SFG in KBBF crystal.

In 2007, our lab ${ }^{7)}$ employ a frequency-tripled Nd:YVO4 laser with a passive-mode-locking technique based on a saturable Bragg reflector as the pumping source of a $2.1 \mathrm{~mm}$ thick KBBF-PCT with a phase matching angle of 68.6 degree, the high power sixth harmonic generation $(3 \omega+3 \omega)$ of an $\mathrm{Nd}$ :YVO4 laser is obtained at $177.3 \mathrm{~nm}$. For the input power of $3.5 \mathrm{~W}$, the maximum output power is $12.9 \mathrm{~mW}$ in a hermetic chamber filled with $\mathrm{N}_{2}$, and this is the highest output power ever obtained in deep ultraviolet region by means of the direct harmonic generation. Moreover, the output power is not saturated yet and higher power should be obtained if more power density at $355 \mathrm{~nm}$ is applied. The experimental setup and the power curve are shown in Fig. 4. Also, in our lab, ${ }^{7}$ the fourth harmonic generation of a Ti: sapphire laser system (150fs) at wavelength $200 \mathrm{~nm}$ with a high conversion
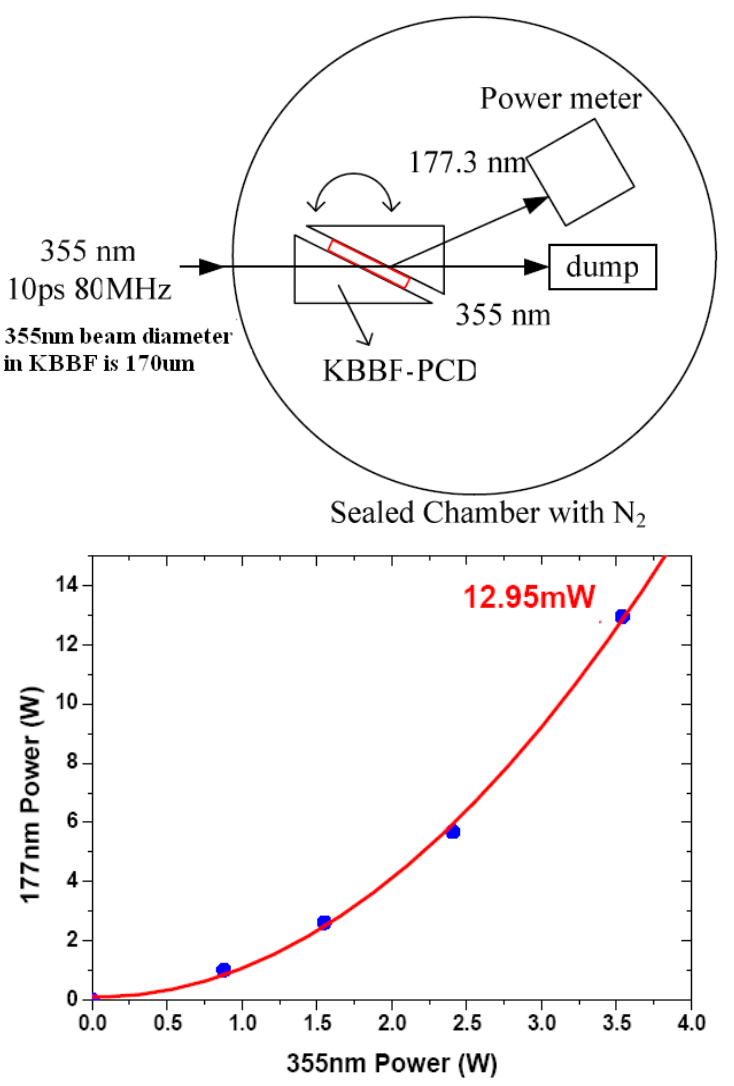

Fig.4 The experiment setup for $12.9 \mathrm{~mW}$ at $177.3 \mathrm{~nm}$ and the output power and in power curse.

efficiency of $26.1 \%$ has been also obtained using the KBBF-PCT with a KBBF size of $10.5 \times 6 \times 2.3 \mathrm{~mm}^{3}$ and a phase matching angle of 55 degree. The maximum output at $200 \mathrm{~nm}$ is $10.7 \mathrm{~mW}$ when the input power at $400 \mathrm{~nm}$ is $40.9 \mathrm{~mW}$. This is the highest conversion efficiency ever obtained in deep ultraviolet region with $\mathrm{KBBF}-\mathrm{CaF}_{2}$ prism-coupled device. Meantime, the conversion efficiency is not saturated yet and higher conversion efficiency could be obtained if higher power intensity at $400 \mathrm{~nm}$ is applied. During the experiment, no damage is found in either $\mathrm{KBBF}$ or $\mathrm{CaF}_{2}$ prisms. The conversion efficiency curve is shown in Fig. 5.

Our $\mathrm{lab}^{7}$ has developed a ns widely tunable t DUV laser in the wavelength range from 175 to $210 \mathrm{~nm}$ by the fourth harmonic generation of Ti:Sapphire laser with KBBF crystal.

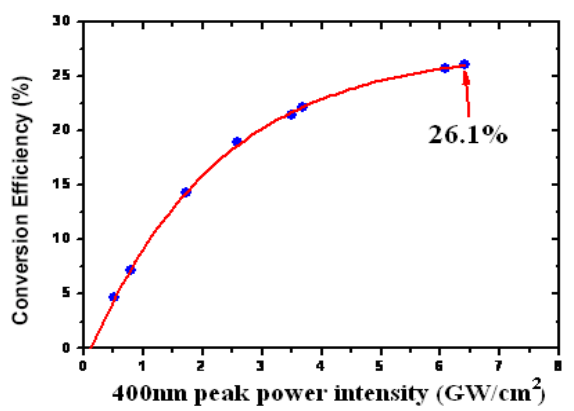

Fig.5 The conversion efficiency curve. 

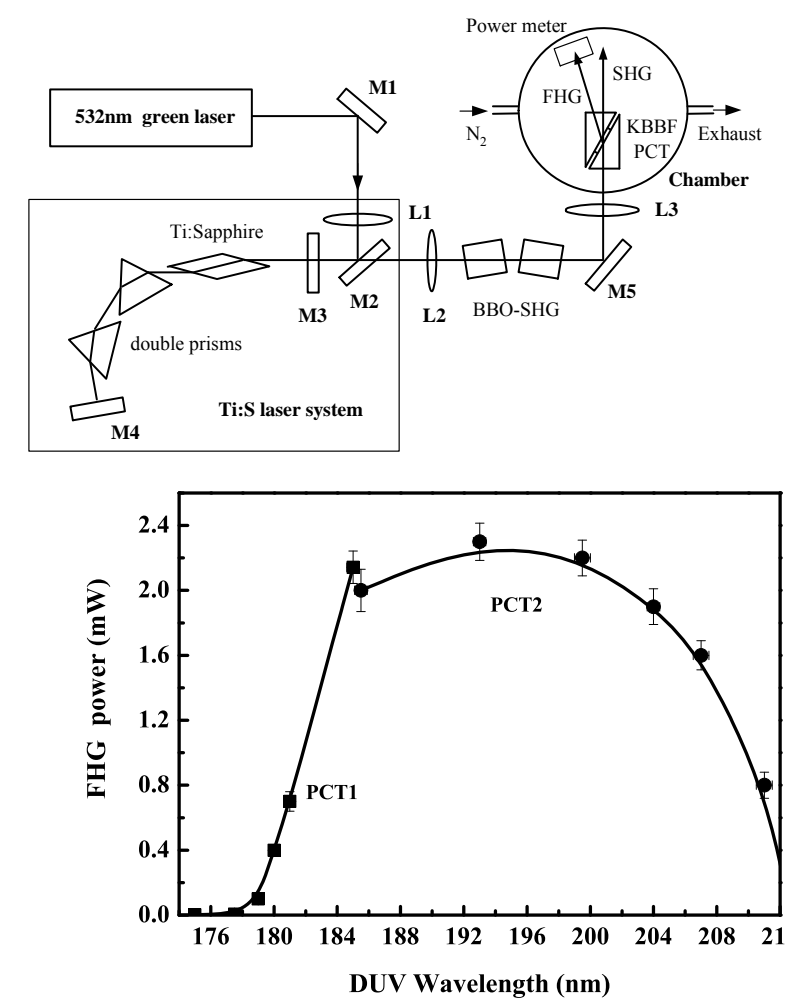

Fig.6 The experiment setup for tunable DUV laser and the tunable wavelength curve.

The highest output power is $2.23 \mathrm{~mW}$ at $193 \mathrm{~nm}$ and the power of the DUV laser is more than $2 \mathrm{~mW}$ from $185 \mathrm{~nm}$ to $200 \mathrm{~nm}$. It is the first demonstration of $\mathrm{mW}$-level ns continuously tunable DUV all-solid-state laser in such a wide wavelength range. The experimental setup and tunable curve are shown in Fig. 6 . The output power of Ti:Sapphire laser is more than $3 \mathrm{~W}$ during the whole tuning range from 690 to $840 \mathrm{~nm}$. At the maximum output power at $780 \mathrm{~nm}$, the beam quality factor is $\mathrm{M}^{2} \sim 3$. The bandwidth (FWHM) is less than $2 \mathrm{~nm}$ and the pulse width (FWHM) is $24 \mathrm{~ns}$. Tunable UV output power higher than $1.5 \mathrm{~W}$ in most tuning range is achieved by this walk-off compensated double BBO configuration. Phase-matching angle of KBBF in type-I SHG changes from $68.3^{\circ}$ to $48.9^{\circ}$ with fundamental wavelength from 345 to $420 \mathrm{~nm}$. Two KBBF PCT devices with thicknesses of $0.65 \mathrm{~mm}$ and $0.69 \mathrm{~mm}$ along $\mathrm{Z}$ axis are used in the wide tunable DUV light generation for the large difference of phase matching angles. One PCT (PCT1) device with KBBF cut at $\theta=66.4^{\circ}$ is best suited for frequency doubling of $354.7 \mathrm{~nm}$ beam to $177.3 \mathrm{~nm}$. The other KBBF PCT device (PCT2) with $\mathrm{KBBF}$ cut at $\theta=56.4^{\circ}$ is optimized for producing 193nm light.

Cooperation with Tokyo university ${ }^{8)}$, an important progress, over $350 \mathrm{~mW}$ DUV laser at $193 \mathrm{~nm}$ and a stable power of $150 \mathrm{~mW}$ are obtained by KBBF crystal, and this is a highest result at $193 \mathrm{~nm}$ by use of the borate crystals. The $\mathrm{KBBF}-\mathrm{CaF}_{2}$ PCT device was also used to generate the fifth harmonic (from 157 to $160 \mathrm{~nm}$ ) of a Ti:sapphire laser system at $1-\mathrm{kHz}$ repetition rate and 16-ns pulse width at $800-785 \mathrm{~nm}$ wavelength range. This process was achieved by

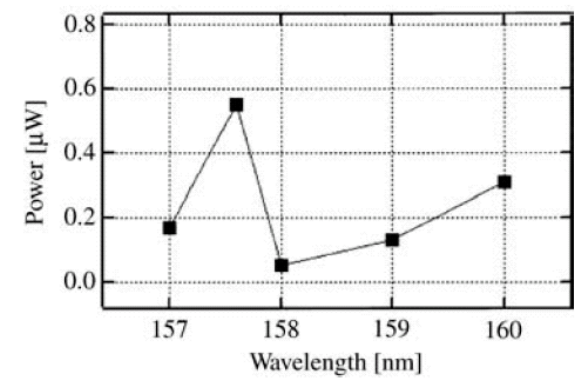

Fig.7 The tunable curve.

sum-frequency mixing of the fourth harmonic with the fundamental $(4 \omega+\omega \rightarrow 5 \omega)$. The $5 \omega$ spectral width is estimated to be $0.01 \mathrm{pm}$ and the pulse width was $9.7 \mathrm{~ns}$ at $157.6 \mathrm{~nm}$. The output power decreased around $158-159 \mathrm{~nm}$, as shown in Fig. 7 , but the reason for this is not clear.

\section{Conclusions and Acknowledgements}

The DUV laser generation in our lab and Tokyo university has obtained great progress in KBBF crystal. The merits of KBBF have been demonstrated through these good results. At last, we believe that the output power and conversion efficiency can be greatly increased in the future if the thicker $\mathrm{KBBF}$ crystal with good optical quality is grown and the application technique of KBBF crystal is further developed. In addition, BPO crystal ${ }^{9)}$ whose characteristics are currently still under investigation has a large transparent range from 130 to $4300 \mathrm{~nm}$ and its birefringence index is 0.0045 . It may be a promising NLO material candidate as DUV laser generation.

This work was supported by the State Key Program for Basic Research of China, the National High Technology Research and Development Program of China, the National Natural Science Foundation of China, and the Knowledge Innovation program of Chinese Academy of Sciences.

\section{References}

1) J. D. Koralek, J. F. Douglas, N. C. Plumb, Z. Sun, A.V. Fedorov, M. M. Murnane, H. C. Kapteyn,S. T. Cundiff, Y. Aiura, K. Oka, H. Eisaki, and D. S. Dessau: Phys. Rew. Lett. 96 (2006) 017005.

2) DY Tang, YN Xia1, BC Wu, C Chen. J. .Crystal Growth. 222 (2001) 125; ZG Hu, M.Yoshimurab, Y. Morib, T. Sasakib: J. .Crystal Growth. 275 (2005) 232.

3) C. Chen, Z. Lin, Z.Wang: Appl. Phys. B. 80 (2005) 1-25. C. Chen: Opt. Mater. 26 (2004) 425.

4) F. Seifert, J. Ringling, F. Noack, V. Petrov, and 0. Kittelmann: Opt. Lett. 19 (1994) 1538.

5) V. Petrov, F. Rotermund and F. Noack: Electron. Lett. 34 (1998) 1748.

6) T. Togashi, T. Kanai, T. Sekikawa, S. Watanabe, C.T. Chen, C.Q. Zhang, Z.Y. Xu, J.Y. Wang: Opt. Lett. 28 (2003) 254.

7) G.L Wang et. al: submitted to Appl. Phys. B. G.L. Wang, et.al submitted to Appl. Opt. HB. Zhang et. al: submitted to Appl.Phys. Lett.

8) T. Kanai, T. Kanda, T. Sekikawa, S. Watanabe, T. Togashi, C.T. Chen, C.Q. Zhang, Z.Y. Xu, J.Y. Wang: J. Opt. Soc. Am. B. 21 (2004) 370 .

9) Z.H. Li, Z.S. Lin, Y.C. Wu, P.Z. Fu, Z.Z. Wang, C. Chen: Chem. Mater. 16 (2004) 2906 